\title{
Performance of the CMS Pixel detector for the Phase I upgrade at HL-LHC
}

\author{
Mark Grimes ${ }^{* \dagger}$ \\ Univ. Bristol \\ E-mail: mark.grimes@bristol.ac.uk
}

\begin{abstract}
The luminosity upgrade of the Large Hadron Collider is foreseen to proceed in two phases. An eventual factor-of-ten increase in LHC statistics will have a major impact in the LHC Physics program. However, the HL-LHC as well as offering the possibility to increase the physics potential will create an extreme operating environment for the detectors, particularly the tracking devices and the trigger system. An increase in the number of minimum-bias events beyond the levels envisioned for design luminosity creates the need to handle much higher occupancies and for the innermost layers unprecedented levels of radiation. This can degrade the performance of the current detector. In order to recover and improve the current level of seeding, tracking, and b-tagging performance an upgrade of the CMS pixel detector system has been proposed for the Phase I of the HL-LHC. Results of Monte Carlo simulation studies for the new pixel detector will be presented and compared to that of the current CMS detector. The upgraded pixel system will provide improved b-tagging, pixel track seeding and stand-alone tracking capabilities, which will be key elements of many CMS physics analyses at the HL-LHC.
\end{abstract}

10th International Conference on Large Scale Applications and Radiation Hardness of Semiconductor Detectors

6-8 July 2011

Florence, Italy

*Speaker.
† On behalf of the CMS Tracker Upgrade Simulations Group 


\section{Introduction}

The CMS detector was originally designed to run at an instantaneous luminosity of $1 \times 10^{34}$ $\mathrm{cm}^{-2} \mathrm{~s}^{-1}[1]$. The LHC is expected to deliver this after a shutdown around 2016, and to double this soon afterwards. How the machine delivers this will be a combination of many factors, including the number of colliding bunches; the number of protons per bunch; and the time between bunch crossings. The combinations of these parameters to achieve a given luminosity alter the mean number of interactions per bunch crossing, known as pileup, which is a significant variable in a detector's ability to reconstruct a trigger event. The extra interactions have to be disentangled from the main physics process of interest, and significantly increase the data rates from the detector. As the level of pile up increases significantly above the design specification, CMS will have to upgrade subdetectors to cope with these issues. The round of upgrades over the 2016 shutdown, known as Phase I, will include an upgrade to the pixel detector.

\section{The current CMS pixel detector}

The pixel detector installed and currently operating in the CMS detector is made from 3 barrel layers and 2 forward discs either side. It is composed from sensor modules with $100 \times 150 \mu \mathrm{m}$ pitch made using $\mathrm{n}^{+}$-on-n technology[2]. The barrel has 48 million pixels[3] and the forward disks 18 million. The geometry is such that the 3 hit coverage is not completely hermetic, with large seeding inefficiencies in the $\eta$ region between 1.5 and 2.5[4].

A severe limitation for high pileup situations is the Read Out Chip (ROC). If the design luminosity is achieved using $25 \mathrm{~ns}$ bunch spacing, the ROC buffer size and readout speed lead to a dynamic inefficiency in the pixel detector of 4\%[4]; with $50 \mathrm{~ns}$ bunch spacing this will be greater than $16 \%[4]$. This inefficiency rises exponentially with luminosity and will significantly reduce the overall tracking performance. Figure 1 shows the degradation from this effect when running at $2 \times 10^{34} \mathrm{~cm}^{-2} \mathrm{~s}^{-1}$ with $25 \mathrm{~ns}$ bunch spacing, equivalent to an average of 50 interactions per crossing. Even without this effect the loss from the lack of 3 hit coverage can be clearly seen around the $\eta 1.5$ region.

The current pixel detector will not survive the radiation damage up to $350 \mathrm{fb}^{-1}$, and at least the inner regions were always intended to be replaced after around $100 \mathrm{fb}^{-1}$ [5] of collected data. The cooling was designed for a readout chip with a higher power draw, adding to a significant amount of passive material that deteriorates performance throughout the whole tracker from multiple scattering, nuclear interactions and photon conversions.

It is clear that the current pixel detector needs to be replaced to run at the high luminosity environment of the LHC beyond 2016. The current detector was designed to be easily removed or installed in a few days. This is a strong advantage, although the replacement will need to use the same readout cabling and service channels. There is a clear opportunity to install an improved detector that can maintain, if not improve, the current low luminosity performance in a much more challenging setting.

\section{The proposed pixel detector upgrade}

The proposed detector has been designed to rectify the shortcomings of the current detector 

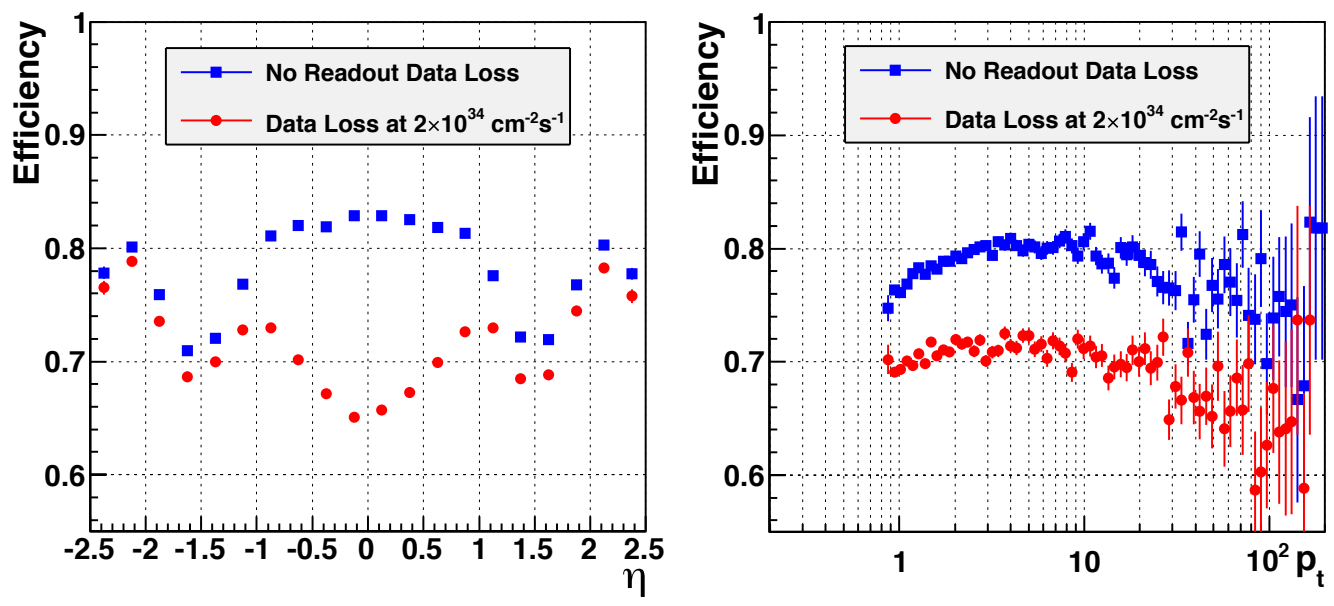

Figure 1: Simulation of the expected tracking efficiency for $t \bar{t}$ events with the current detector at 50 pileup (equivalent to $2 \times 10^{34} \mathrm{~cm}^{-2} \mathrm{~s}^{-1}$ ) versus eta (left) and versus transverse momentum (right). The efficiency with no data loss is shown in blue, and the efficiency with the estimated data loss from buffer overflows is shown in red[4].

in the more challenging high luminosity environment. There is some ongoing $R \& D$ to establish other radiation hard technologies, but the baseline design uses the same $\mathrm{n}^{+}$-on- $\mathrm{n}$ technology as the current detector. The pixel pitch is also being kept at $100 \times 150 \mu \mathrm{m}$. There are however some very significant changes.

A fourth barrel layer and an extra disc either side have been added to help with pattern recognition and improve standalone tracking efficiency. The innermost barrel layer has also been moved closer to the beam line to improve impact parameter resolution and $b$ tagging. Figure 2 shows the layout of the old and new geometries.
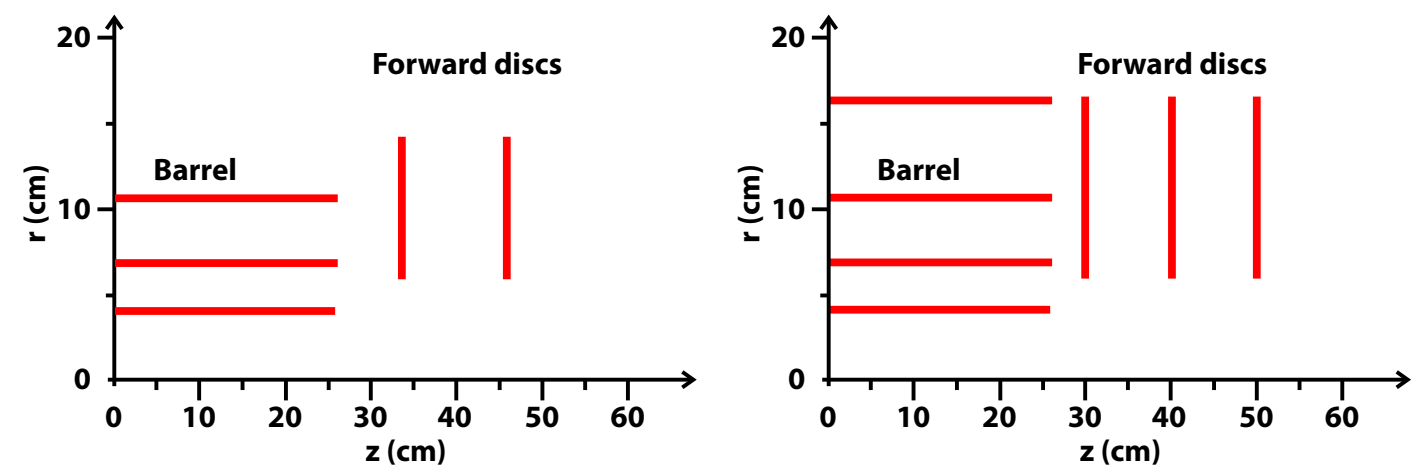

Figure 2: The geometry of the proposed pixel detector upgrade (right), compared to the current geometry (left)[5].

The amount of passive material has been significantly reduced by moving readout electronics and connectors further out. Bi-phase $\mathrm{CO}_{2}$ cooling will also replace the $\mathrm{C}_{6} \mathrm{~F}_{14}$ single phase cooling currently used, allowing smaller heat exchanger pads and pipes[4].

The current ROC is sufficiently radiation hard for the LHC environment, and is well understood, but as previously stated cannot cope with the required data rates. The replacement ROC 
will be based on the current design but with some important changes. Larger data buffers will be included to cope with the data rates, and an additional buffer stage will be required to store the Level 1 trigger verified hits until a readout token arrives. The readout speed will need to be increased, since a larger number of modules will have to use the same number of optical readout fibres. To achieve this a digital readout using on chip ADC will be implemented running at 160 $\mathrm{MHz}$, compared to the current $40 \mathrm{MHz}$ analogue readout.

\section{Simulation setup and results}

To estimate the performance of the current and upgraded pixel detectors at high luminosities, full GEANT4 based simulations have been run. The simulations for the current geometry use triplet track seeding, and quadruplet and triplet for the upgraded geometry. Figure 3 shows the performance of the old and upgraded detectors for $t \bar{t}$ events at 50 pile up (equivalent to $2 \times 10^{34}$ $\mathrm{cm}^{-2} \mathrm{~s}^{-1}$ with $25 \mathrm{~ns}$ bunch spacing). There is a clear improvement in both the efficiency (the fraction of signal tracks found) and the fakerate (the fraction of reconstructed tracks that are created from unrelated hits).
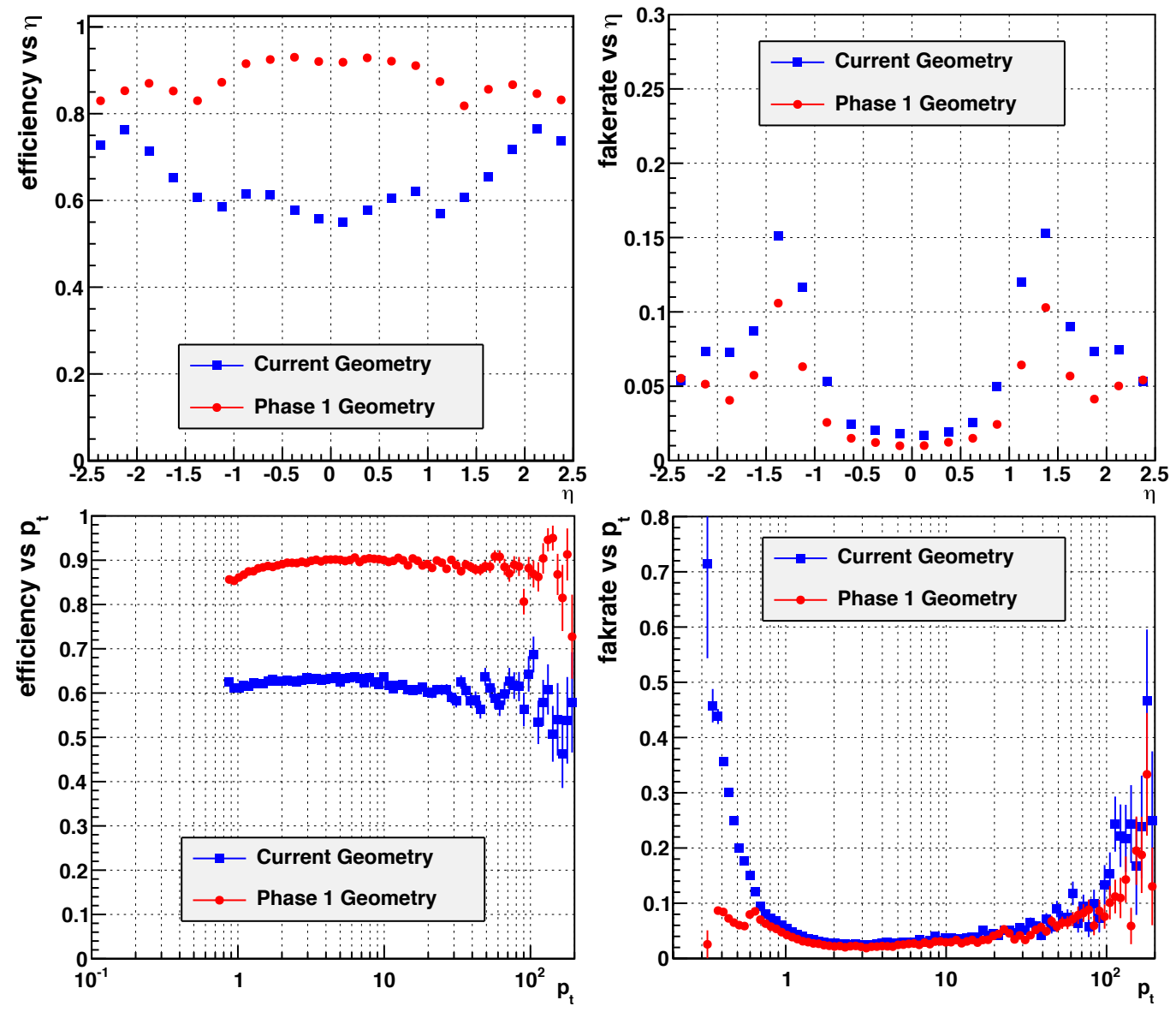

Figure 3: The reconstruction performance of simulated $t \bar{t}$ events at 50 pileup using the current pixel detector (blue), and the upgraded pixel detector(red)[4]. Note that figure 1 uses low purity track cuts, whereas high purity track cuts are used here. 
A number of important physics analyses involve final states with high energy isolated muons. Muon identification and reconstruction is therefore an important goal of CMS, and indeed drove a number of the original design decisions. Figure 4 shows the reconstruction performance at 50 pileup, where the current detector has a $15 \%$ efficiency drop compared to the upgraded detector over the full transverse momentum range. Analyses requiring two well reconstructed muons would therefore suffer a $(0.85)^{2}$ loss in efficiency compared to using the upgraded pixel detector.
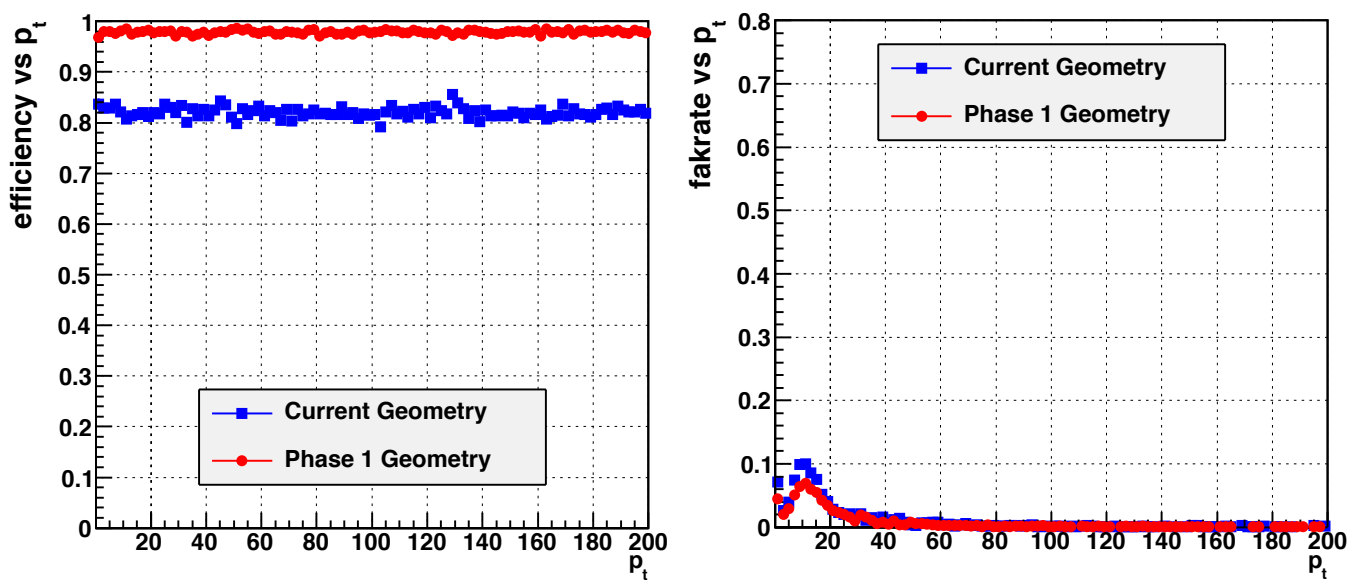

Figure 4: Reconstruction performance for simulated single muons with a range of energies at 50 pileup[4].

Good impact parameter resolution is required for calculating the positions of primary and secondary vertices, which is in turn important for identifying longer lived particles. Figure 5 shows the impact parameter resolution in the barrel region for the current and upgraded detector. Results for other regions are available in [4] and show similar improvement.

Reconstructing vertices is one of the main requirements of the pixel detector. At higher pileup, it is important to separate the interesting trigger event from superimposed coincident soft interactions (i.e. the pileup events). Doing so will require efficient vertex reconstruction, and correct association of tracks to these vertices. Figure 6 shows the resolution of the reconstructed primary vertex as a function of the number of tracks. The upgraded detector shows an improvement at 50 pileup over the current detector with no pileup.

Many interesting physics channels have final states with $b$ jets, and so reliably tagging jets that come from a $b$ quark is vital for studying these channels. Figure 7 shows the $b$ tagging performance with no pileup and 50 pileup. At high pileup the upgraded detector can be seen to almost regain the performance of the current detector at 0 pileup.

\section{Summary and outlook}

The CMS upgrade community has drafted a credible design for a replacement pixel detector that will perform through the envisaged LHC operation to 2020. For the sensitive material conservative technologies have been used in the baseline design, with production methods already proven for the current detector. Flexibility on the final design choice has been kept however, should R\&D into improved technologies prove fruitful on the required timescale. For other areas, such as the bi-phase $\mathrm{CO}_{2}$ cooling, the $\mathrm{R} \& \mathrm{D}$ program is well established[4]. 

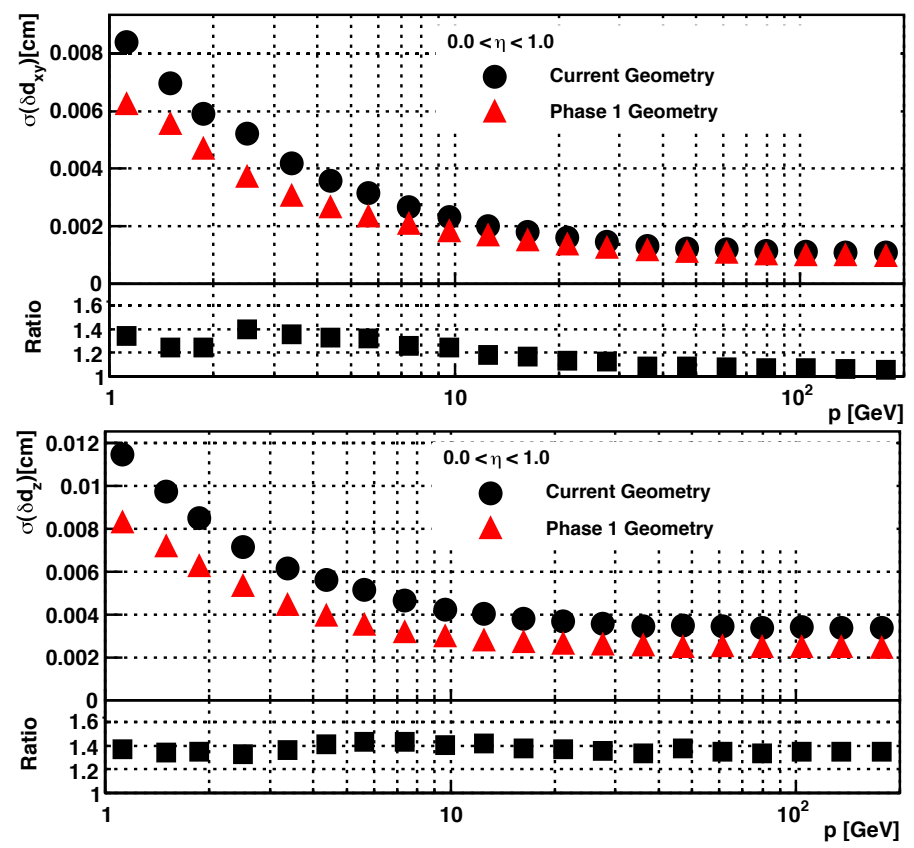

Figure 5: The transverse (top) and longitudinal (bottom) impact parameter resolutions versus transverse momentum in the barrel region [4]. The ratio of the current geometry resolution divided by the upgraded geometry resolution is also shown to illustrate the relative improvement.

Detailed Monte Carlo simulations have shown that the upgraded performance at high pileup will be as good, if not better, than the current detector without pileup. Preliminary steps have been taken to show that the detector performance carries through to improvements in the physics performance[4]. In the near future these steps will be expanded to complete analyses on simulated data, to fully justify the upgrade cost and show that the CMS physics program can continue unabated through to 2020 .

\section{References}

[1] CMS Collaboration, CMS Physics Technical Design Report Volume I : Detector Performance and Software, CERN-LHCC-2006-001. CMS-TDR-008-1 (2006)

[2] G. Bolla, D. Bortoletto, R. Horisberger, R. Kaufmann, T. Rohe, A. Roy, Sensor development for the CMS pixel detector, Nucl. Instrum. Meth. A485 (2002) 89-99

[3] A. Dominguez (for the CMS Pixel Group), The CMS pixel detector, Nucl. Instrum. Meth. , A581 (2007) 343-346

[4] CMS Collaboration, Technical Proposal for the Upgrade of the CMS detector through 2020, CERN-LHCC-2011-006. LHCC-P-004 (2011)

[5] K. Gill (for the CMS Collaboration), Upgrade of the CMS tracker, Nucl. Instrum. Meth. A636 (2011) 194-200

[6] C. Weiser, A Combined Secondary Vertex Based B-Tagging Algorithm in CMS, CERN-CMS-NOTE-2006-014 

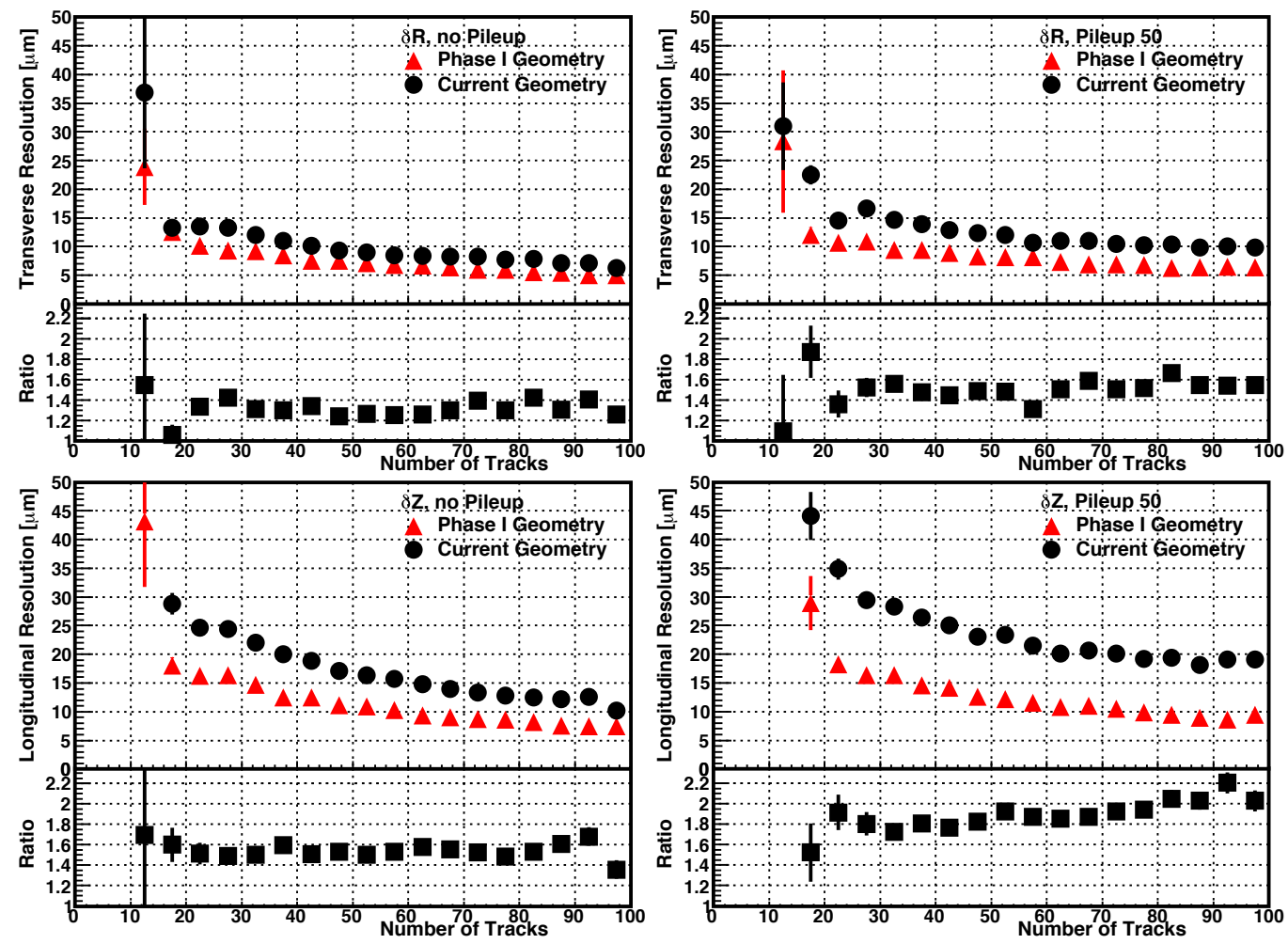

Figure 6: The transverse (top two) and longitudinal (bottom two) primary vertex resolutions at 0 pileup (left two) and 50 pileup (right two)[4]. The ratio of the two resolutions is also plotted to illustrate the relative improvement.
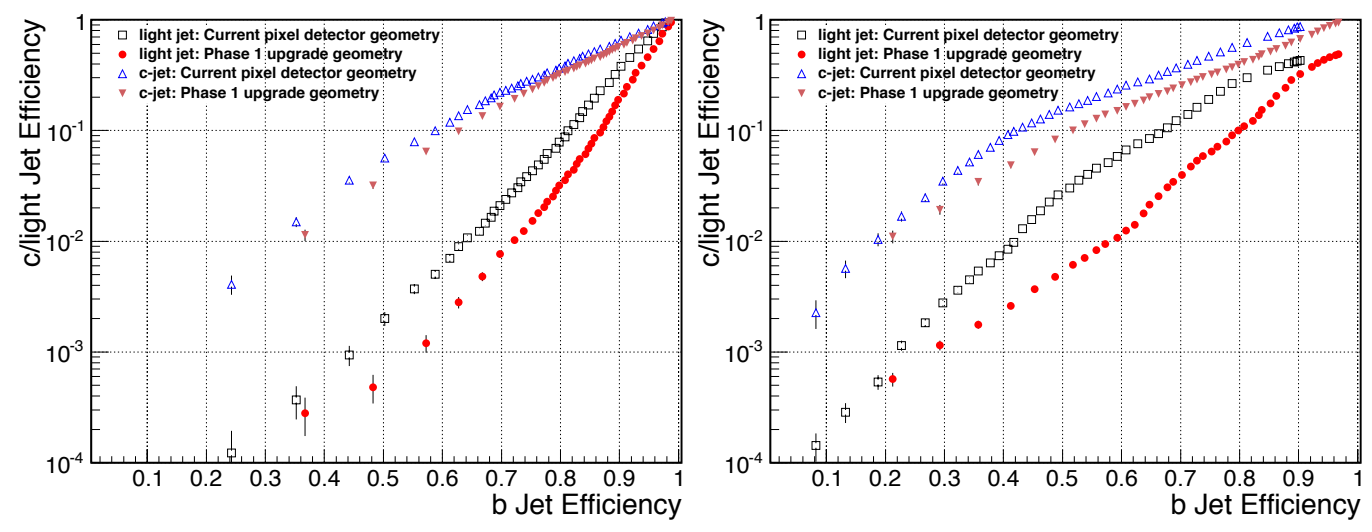

Figure 7: The $b$ tagging performance at 0 pileup (left) and 50 pileup (right)[4]. The $x$ axis shows the efficiency for successfully tagging the simulated $b$ jets, while the $y$ axis shows the efficiency for incorrectly tagging $u, d, s$ or $c$ jets as $b$ jets. Hence a point in the bottom right will show a very efficient and pure $b$ tag. The Combined Secondary Vertex Algorithm[6] has been used. 